\title{
SHAKEDOWN AND COLLAPSE OF ELASTIC-PLASTIC STRUCTURES UNDER VARIABLE AND CYCLIC LOADS
}

\author{
Pham Duc Chinh \\ Institute of Mechanics, 264 Doi Can, Hanoi
}

\begin{abstract}
The paper presents an introduction to the results obtained mainly by the author in shakedown analysis of elastic plastic structures, with the shakedown theorems for elastic plastic kinematic hardening materials playing the central role. Some illustrative examples of application are given.
\end{abstract}

\section{INTRODUCTION}

Plastic collapse, along with Euler elastic instability of geometrically-thin structural members and Griffiths critical crack fracture of brittle-like solids, are among the outstanding phenomena limiting the load-bearing capacity of structures. Attainment of the ultimate yield state at a point within a structure does not imply plastic collapse but that at entire sections to make the structure a mechanism, which could not sustain the larger loads, does. Plastic instantaneous collapse of structures is determined by the static and kinematic theorems of plastic limit analysis. A structure subjected to loads varying arbitrarily within certain limits may fails even at lower load limits than those set by the instantaneous collapse criterion because the plastic deformations would accumulate unrestrictively or be bounded but vary cyclically and unceasingly. Otherwise, the total plastic dissipation in the structure would be bounded, hence its overall response to the external agencies should shake down to some purely elastic state due to a finite residual stress field developed inside the structure. Classical shakedown theory for elastic-perfectly plastic bodies has been well established in the literature. As for the plastic limit theory, the essential contents of the classical shakedown theory are its dual static and kinematic theorems, which are path-independent (loading-history-independent): both theorems determine the shakedown boundary in the loading space under which a loaded structure should be safe regardless of the loading history, while it should fail if the boundary is allowed to be violated unlimitedly. The shakedown design is not only safer its limiting case - the plastic limit one, but also applies to the larger class of dynamic problems, which lie outside the reach of the plastic limit theorems. In fact, under dynamic loading, a structure would never fail instantaneously, thank to the inertia effect, but fail incrementally (monotonically or nonmonotically) or plastic-cyclically with time. 


\section{SHAKEDOWN THEOREMS}

Originally Melan-Koiter shakedown theorems have been constructed for idealistic elastic perfectly plastic material model, which is practical and suffice for plastic limit analysis. For shakedown analysis, much more realistic and practical material model is the elastic plastic kinematic hardening one involving Bauschinger effect. The hardening curve is generally nonlinear, plastic-deformation-history dependent, and is bounded by the initial and ultimate yield stresses. Shakedown static and kinematic theorems have been constructed for the kinematic hardening material satisfying a realistic positive hysteresis postulate, which do not involve the plastic-deformation-history dependent hardening curve, except the initial yield stress and ultimate yield strength, keeping the path-independent spirit of classical Melan-Koiter theorems (Pham, 2007, 2008).

Let $\boldsymbol{\sigma}^{e}(\mathbf{x}, t)$ denote the fictitious elastic stress response of the body $V$ (under the assumption of its perfectly elastic behaviour) to external agencies over a period of time $(\mathbf{x} \in V, t \in[0, T])$, called a loading history. The actions of all kinds of external agencies upon $V$ can be expressed explicitly through $\boldsymbol{\sigma}^{e}$. At every point $\mathbf{x} \in V$, the elastic stress response $\boldsymbol{\sigma}^{e}(\mathbf{x}, t)$ is confined to a bounded time-independent domain with prescribed limits in the stress space, called a local loading domain $\mathcal{L}_{x}$. As a field over $V, \boldsymbol{\sigma}^{e}(\mathbf{x}, t)$ belongs to the time-independent global loading domain $\mathcal{L}$ :

$$
\mathcal{L}=\left\{\boldsymbol{\sigma}^{e} \mid \boldsymbol{\sigma}^{e}(\mathbf{x}, t) \in \mathcal{L}_{x}, \mathbf{x} \in V, t \in[0, T]\right\} .
$$

In the spirit of shakedown theorems, the bounded loading domain $\mathcal{L}$, instead of a particular loading history $\boldsymbol{\sigma}^{e}(\mathbf{x}, t)$, is given a priori. Shakedown of a body in $\mathcal{L}$ means it shakes down for all possible loading histories $\boldsymbol{\sigma}^{e}(\mathbf{x}, t) \in \mathcal{L}$. $k_{s}$ denotes the shakedown safety factor: at $k_{s}>1$ the structure will shake down, while it will not at $k_{s}<1$, and $k_{s}=1$ defines the boundary of the shakedown domain.

\section{Shakedown static theorem}

$$
k_{s}=\min \{\bar{U}, \bar{C}\}
$$

where

$$
\begin{aligned}
& \bar{U}=\sup _{\boldsymbol{\rho} \in \mathcal{R}}\left\{k \mid k\left(\boldsymbol{\rho}+\boldsymbol{\sigma}^{e}\right) \in \mathcal{Y}_{u}, \forall \boldsymbol{\sigma}^{e} \in \mathcal{L}\right\}, \\
& \bar{C}=\sup _{\boldsymbol{\rho}^{\prime}}\left\{k \mid k\left(\boldsymbol{\rho}^{\prime}+\boldsymbol{\sigma}^{e}\right) \in \mathcal{Y}_{i}, \forall \boldsymbol{\sigma}^{e} \in \mathcal{L}\right\},
\end{aligned}
$$

$\mathcal{R}$ is the set of admissible time-independent self-equilibrated residual stress fields $\boldsymbol{\rho}(\mathbf{x})$ that satisfy homogeneous equilibrium equations on $V ; \boldsymbol{\rho}^{\prime}$ is a time-independent stress field that is not requied to be self-equilibrated; $\mathcal{Y}_{u}$ designates the elastic domain in the stress space that is bounded by the yield surface determined by the ultimate yield stress $\sigma_{Y}^{u}$, while $\mathcal{Y}_{i}$ is the respective domain bounded by the yield surface determined by the initial yield stress $\sigma_{Y}^{i}$.

\section{Shakedown kinematic theorem}

$$
k_{s}^{-1}=\max \{U, C\},
$$


where

$$
\begin{gathered}
U=\sup _{\mathbf{e}^{p} \in \mathcal{A} ; \boldsymbol{\sigma}^{e} \in \mathcal{L}} \frac{\int_{0}^{T} d t \int_{V} \boldsymbol{\sigma}^{e}: \mathbf{e}^{p} d V}{\int_{0}^{T} d t \int_{V} D_{u}\left(\mathbf{e}^{p}\right) d V}, \\
C=\sup _{\mathbf{x} \in V ; \boldsymbol{\sigma}^{e} \in \mathcal{L} ; \hat{\mathbf{e}}^{p} ; \boldsymbol{\rho}^{\prime}} \frac{\left(\boldsymbol{\sigma}^{e}+\boldsymbol{\rho}^{\prime}\right): \hat{\mathbf{e}}^{p}}{D_{i}\left(\hat{\mathbf{e}}^{p}\right)},
\end{gathered}
$$

$\mathcal{A}$ is the set of compatible-end-cycle plastic strain rate fields $\mathbf{e}^{p}$ over the time cycles $0 \leq t \leq T$ :

$$
\mathcal{A}=\left\{\mathbf{e}^{p} \mid \varepsilon^{p}=\int_{0}^{T} \mathbf{e}^{p} d t \in \mathcal{C}\right\}
$$

$\mathcal{C}$ is the set of compatible plastic strain increment fields on $V ; \hat{\mathbf{e}}^{p}$ and $\boldsymbol{\rho}^{\prime}$ are plastic strain rate and time-independent stress fields that are not required to satisfy any compatibility and equilibrium constraints; $D\left(\mathbf{e}^{p}\right)$ is the dissipation function determined by the yield stress $\sigma_{Y}$ and the respective yield criterion, e.g. for a Mises material

$$
D\left(\mathbf{e}^{p}\right)=\sqrt{2 / 3} \sigma_{Y}\left(\mathbf{e}^{p}: \mathbf{e}^{p}\right)^{1 / 2},
$$

while for a Tresca material:

$$
D\left(\mathbf{e}^{p}\right)=\frac{1}{2} \sigma_{Y}\left(\left|e_{1}^{p}\right|+\left|e_{2}^{p}\right|+\left|e_{3}^{p}\right|\right)=\sigma_{Y} \max \left\{\left|e_{1}^{p}\right|,\left|e_{2}^{p}\right|,\left|e_{3}^{p}\right|\right\},
$$

$e_{1}^{p}, e_{2}^{p}, e_{3}^{p}$ are the principal plastic strain rates; $D_{u}\left(\mathbf{e}^{p}\right)$ and $D_{i}\left(\mathbf{e}^{p}\right)$ are the dissipation functions with $\sigma_{Y}^{u}$ and $\sigma_{Y}^{i}$ taking the places of $\sigma_{Y}$, respectively.

At $\bar{U}>\bar{C}$ of criterion (2) [or $U<C$ of criterion (5)] the nonshakedown collapse mode is cyclic, otherwise the incremental collapse mode prevails.

For broad classes of problems, the following reduced kinematic theorem is useful (Pham and Stumpf, 1994; Pham, 2008)

\section{Reduced kinematic theorem}

$$
k_{s}^{-1}=\max \{I, A\},
$$

where

$$
\begin{gathered}
I=\sup _{\boldsymbol{\sigma}^{e} \in \mathcal{L} ; \boldsymbol{\varepsilon}^{p} \in \mathcal{C}} \frac{\int_{V} \max _{t_{x}}\left[\boldsymbol{\sigma}^{e}\left(\mathbf{x}, t_{x}\right): \boldsymbol{\varepsilon}^{p}(\mathbf{x})\right] d V}{\int_{V} D_{u}\left(\boldsymbol{\varepsilon}^{p}\right) d V} \\
A=\sup _{\mathbf{x} \in V ; \boldsymbol{\sigma}^{e} \in \mathcal{L} ; \hat{\boldsymbol{\varepsilon}}^{p} ; t_{1}, t_{2}} \frac{\left[\boldsymbol{\sigma}^{e}\left(\mathbf{x}, t_{1}\right)-\boldsymbol{\sigma}^{e}\left(\mathbf{x}, t_{2}\right)\right]: \hat{\boldsymbol{\varepsilon}}^{p}(\mathbf{x})}{2 D_{i}\left(\hat{\boldsymbol{\varepsilon}}^{p}\right)} .
\end{gathered}
$$




\section{SOME ILLUSTRATIVE EXAMPLES}

Aa a first example of application, let us consider a beam of rectangular cross-section of the size $b \times 2 h$, which is bended up and down alternatively with the moment $\pm M$ (fig. 1 ). The outermost layers on the upper and lower sides of the beam begin to yield at the moment $M=M_{Y}^{i}=\sigma_{Y}^{i} \frac{2}{3} b h^{2}$, while the ultimate yielding moment (the plastic limit load) of the beam is $M=M_{Y}^{u}=\sigma_{Y}^{u} b h^{2}$. Application of eqs. (11)-(13) gives the obvious result:

$$
k_{s}^{-1}=\max \{I, A\}=\max \left\{M / M_{Y}^{u}, M / M_{Y}^{i}\right\}=M / M_{Y}^{i}
$$

hence at $M=M_{Y}^{i}\left(k_{s}=1\right)$, the beam fails because of alternating plasticity started from the failure of the beam's outermost layers.

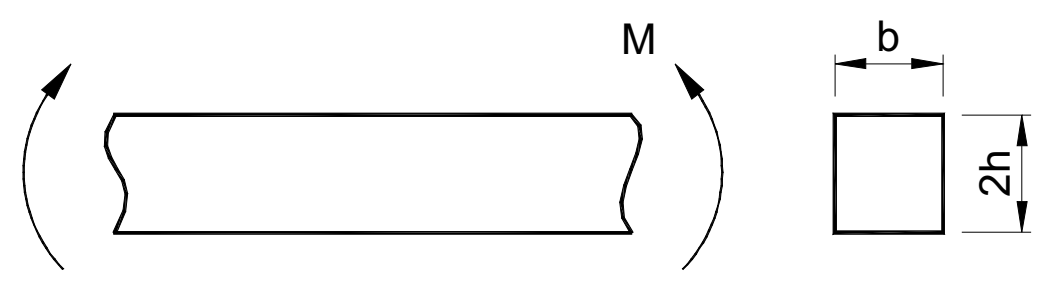

Fig. 1. A beam in bending

Next, consider a beam clamped at the two ends $A$ and $D$ (no horizontal kinematic constraint). The vertical point load $P$ is applied and removed slowly, but alternatively, at $B$ and then $C$ for un unlimited number of times. Application of criterion (12) with an optimal incremental collapse mechanism ABC shown in fig. 2 yields the nonshakedown limit load: $P_{S D}=8.1 M_{Y}^{u} / l$ (the respective plastic limit load is significantly larger: $P_{P}=9 M_{Y}^{u} / l$ ).
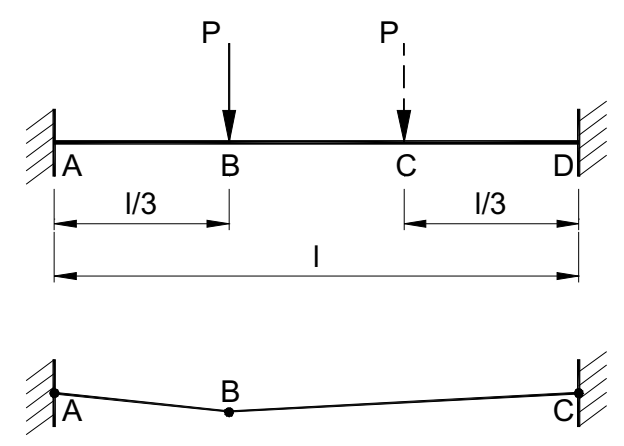

Fig. 2. A clamped beam under sequential loads

A thick-walled hollow sphere of inner and outer radii $a$ and $b$ is subjected to quasistatic internal pressure $q$, which may vary arbitrarily from 0 to the limit $q^{U}$ (fig. 3 ). Application of criterion (11)-(13) yields

$$
k_{s}^{-1}=\max \{I, A\}=q^{U} \max \left\{\frac{1}{2 \sigma_{Y}^{u} \ln (b / a)}, \frac{3 b^{3}}{4 \sigma_{Y}^{i}\left(b^{3}-a^{3}\right)}\right\} .
$$




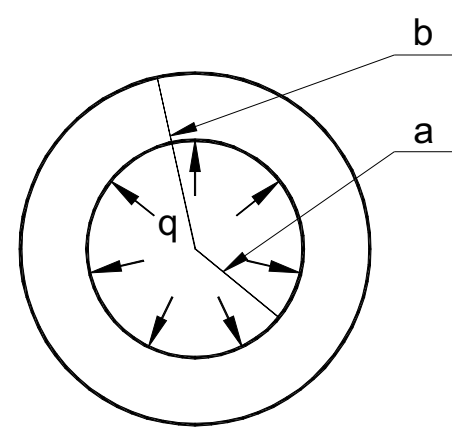

Fig. 3. A hollow sphere under variable pressure

In this case, the incremental collapse load coincides with the plastic limit one, while the alternating plasticity happens at the inner radius of the hollow sphere.

At last consider a disk of radius $a$ clamped at the edge and subjected to uniform quasiperiodic dynamic pressure

$$
q=q_{0}+q_{1} \sin \omega t
$$

where $q_{0}=$ const, $q_{1}$ and $\omega$ are quasistatic functions of time varying between the limits

$$
0 \leq q_{1} \leq q_{1}^{U}, \quad 0 \leq \omega \leq \omega_{U}<\omega_{I},
$$

$\omega_{I}$ is the principal natural frequency of the structure. Denote

$$
k^{4}=\frac{h m}{D} \omega^{2}, \quad k_{U}^{4}=\frac{h m}{D} \omega_{U}^{2}, \quad D=\frac{E h^{3}}{12\left(1-\nu^{2}\right)},
$$

where $E$ is the Young's modulus, $\nu$ - the Poisson ratio, $m$ - the mass density, and $h$ is the thickness of the disk.

From the result for the elastic perfectly plastic material (Pham, 1997), we deduce the corresponding result for kinematic hardening material

$$
k_{s}^{-1}=\max \{I, A\},
$$

where

$$
\begin{gathered}
I=\frac{a^{2}}{12 M_{Y}^{u}}+\frac{q_{1}^{U}}{2 M_{Y}^{u}}\left|P_{r}\left(k_{U}, a\right)\right|+\frac{q_{1}^{U}}{2 M_{Y}^{u} a} \int_{0}^{a}\left|P_{\theta}\left(k_{U}, r\right)\right| d r \\
A=\frac{3 q_{1}^{U}}{2 M_{Y}^{i}}\left|P_{r}\left(k_{U}, a\right)\right|
\end{gathered}
$$

(with $A=1$, the alternating plasticity collapse mode happens at the disk's perimeter),

$$
M_{Y}^{u}=\frac{h^{2}}{4} \sigma_{Y}^{u}, \quad M_{Y}^{i}=\frac{h^{2}}{4} \sigma_{Y}^{i},
$$




$$
\begin{aligned}
P_{r}(k, r) & =\left[k I_{1}(k a) J_{0}(k r)-k J_{1}(k a) I_{0}(k r)-\frac{1-\nu}{r} I_{1}(k a) J_{1}(k r)\right. \\
& \left.+\frac{1-\nu}{r} J_{1}(k a) I_{1}(k r)\right] k^{-3}\left[J_{0}(k a) I_{1}(k a)+J_{1}(k a) I_{0}(k a)\right]^{-1}, \\
P_{\theta}(k, r) & =\left[\nu k I_{1}(k a) J_{0}(k r)-\nu k J_{1}(k a) I_{0}(k r)+\frac{1-\nu}{r} I_{1}(k a) J_{1}(k r)\right. \\
& \left.-\frac{1-\nu}{r} J_{1}(k a) I_{1}(k r)\right] k^{-3}\left[J_{0}(k a) I_{1}(k a)+J_{1}(k a) I_{0}(k a)\right]^{-1},
\end{aligned}
$$

$J_{0}, J_{1}, I_{0}, I_{1}$ are Bessel functions.

As an illustration, we fix the value $Q_{0}=q_{0} \frac{a^{2}}{2 M_{Y}^{u}}=4.6$. A graphical display of the incremental collapse curve $I=1$ and the alternating plasticity collapse ones $A=1$ in the plane of dimensionless coordinates $Q_{1}=q_{1}^{U} \frac{a^{2}}{2 M_{Y}^{u}}$ against $K=k_{u} a$, at various values of $\sigma_{Y}^{i} / \sigma_{Y}^{u}=1 / 1.5,1 / 2,1 / 3$ (corresponding in order to, presumably, increasing numbers of loading cycles; presume $\sigma_{Y}^{u}$ is fixed for the material) are presented in Fig. 4. The domain under both the curves $I=1$ and $A=1$, is the shakedown domain. At high numbers of cycles, $\sigma_{Y}^{i}$ should be lowered toward the fatigue limit and the shakedown domain would be reduced correspondingly. With $\sigma_{Y}^{i} / \sigma_{Y}^{u}=1 / 2$, the collapse mode changes from the alternating plasticity one to the incremental one as $K$ increases (at about $K=2.2$ ). With $\sigma_{Y}^{i} / \sigma_{Y}^{u}=1 / 1.5$, the disk fails only incrementally, while with $\sigma_{Y}^{i} / \sigma_{Y}^{u}=1 / 3$, the alternating plasticity mode is the one that prevails.

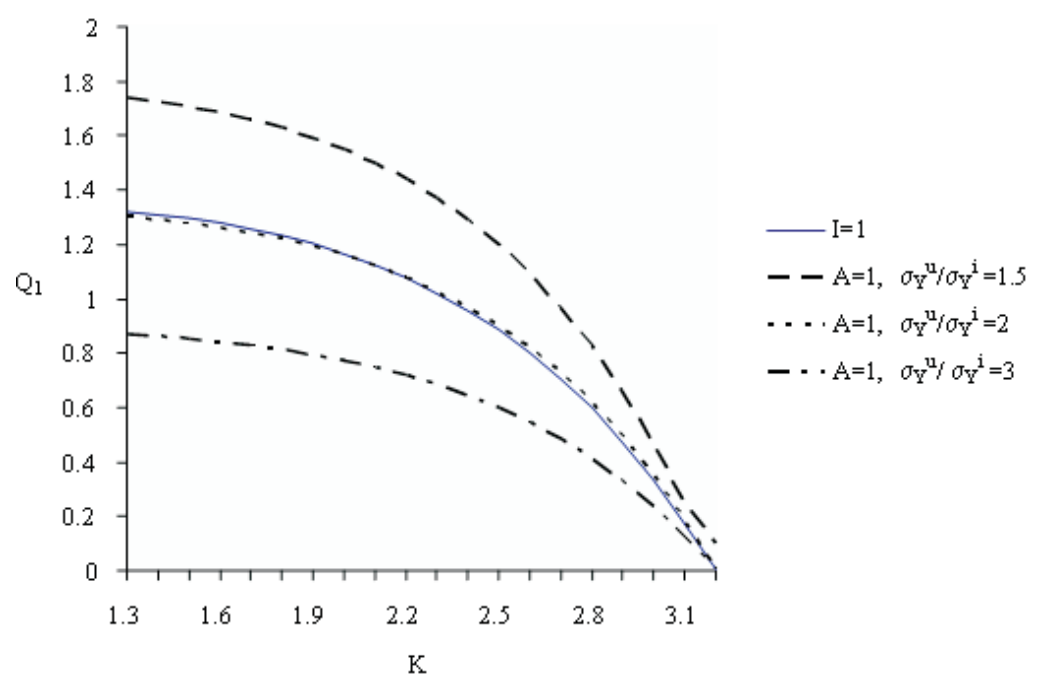

Fig. 4. Incremental $(I=1)$ and alternaning plasticity $(A=1)$ collapse curves at various values of $\sigma_{Y}^{u} / \sigma_{Y}^{i}$, in the plane of dimensionless load amplitude $Q_{1}$ and frequency $K$ 


\section{CLOSURE}

For more details about our results and application of the theorems in solving many practical engineering problems (mainly on elastic perfectly plastic structures) see the References.

Future directions:

- Develope methods to solve problems for practical engineering structures made of realistic kinematic hardening materials, especially those subjected to dynamic loading.

- Improve further the base of shakedown theory, and extend the shakedown analysis to various complicated material models.

\section{ACKNOWLEDGEMENTS}

The work is supported by the National Science Foundation of Vietnam.

This is the content of a plenary report presented at the National Conference on Mechanics commemorizing the 30-th anniversary of the Institute of Mechanics and Vietnam Journal of Mechanics, Hanoi 8/4/2009.

\section{REFERENCES}

[1] Pham D.C., Extended shakedown theorems for elastic-plastic bodies under quasi-periodic dynamic loading. Proceedings of the Royal Society of London A 439 (1992) 649-658.

[2] Pham D.C., Shakedown of bars subjected to cycles of loads and temperature. International Journal of Solids and Structures 30 (1993) 1173-1179.

[3] Pham D.C., Stumpf H., Kinematical approach to the shakedown analysis of some structures. Quarterly of Applied Mathematics 52 (1994) 707-719.

[4] Pham D.C., Adaptation of spherical and cylindrical vessels to variable internal pressure and temperature. International Journal of Mechanical Sciences 37 (1995) 783-792.

[5] Pham D.C., Dynamic shakedown and a reduced kinematic theorem. International Journal of Plasticity 12, 1055-1068 (1996)

[6] Pham D.C., Shakedown analysis for trusses and frames. ASME Journal of Applied Mechanics 64 (1997) 415-419.

[7] Pham D.C., Evaluation of shakedown loads for plates. International Journal of Mechanical Sciences 39 (1997) 1415-1422.

[8] Pham D.C., Plastic failure of reinforced plates under loading cycles. European Journal of Mechanics, A/Solids 16 (1997) 1011-1023.

[9] Pham D.C., Reduced forms of shakedown kinematic theorem for elastic-perfectly plastic bodies. Proceedings of the Royal Society of London A 453 (1997) 2259-2269.

[10] Pham D.C., Failure of a circular reinforced concrete plate against dynamic fluctuating loads. Archive of Applied Mechanics 68 (1998) 711-718.

[11] Pham D.C., Shakedown limits for reinforced beam structures under fluctuating loads. International Journal of Solids and Structures 36 (1999), 1297-1309.

[12] Pham D.C., On dynamic cycle collapse of circular plates. ASME Journal of Applied Mechanics 66 (1999) 250-253.

[13] Pham D.C., An upper bound kinematic approach to the shakedown analysis of structures. Meccanica 34 (1999) 49-56.

[14] Pham D.C., Safety and collapse of elastic-plastic beams against dynamic loads. International Journal of Mechanical Sciences 42 (2000) 575-592. 
[15] Pham D.C., From local failure toward global collapse of elastic plastic structures in fluctuating fields. International Journal of Mechanical Sciences 42 (2000) 819-829.

[16] Pham D.C., Dynamic nonshakedown collapse of simply surported rectangular plate. Zeitschrift für Angewante Mathematik und Mechanik 80 (2000) 283-288.

[17] Pham D.C., Non-shakedown collapse of a doubly-reinforced rectangular plate under cyclic loads. European Journal of Mechanics, A/Solids 19 (2000) 795-810.

[18] Pham D.C., Shakedown kinematic theorem for elastic-perfectly plastic bodies. International Journal of Plasticity 17 (2001) 773-780.

[19] Pham D.C., Weichert D., Shakedown analysis for elastic plastic bodies with limited kinematic hardening. Proceedings of the Royal Society of London A 457 (2001) 1097-1110.

[20] Nguyen Q.S., Pham D.C., On shakedown theorems in hardening plasticity. Compte Rendus Mecanique 329 (2001) 307-314.

[21] Pham D.C., Load bearing capacity of reinforced concrete beams subjected to dynamic cyclic loads. ASCE Journal of Engineering Mechanics 127 (2001) 955-958.

[22] Pham D.C., Plastic collapse of a circular plate under cyclic loads. International Journal of Plasticity 19 (2003) 547-559.

[23] Pham D.C., Shakedown theory for elastic-perfectly plastic bodies revisited. International Journal of Mechanical Sciences 45 (2003) 1011-1027.

[24] Pham D.C., Shakedown static and kinematic theorems for elastic-plastic limited linear kinematic hardening solids. European Journal of Mechanics, A/Solids 24 (2005) 35-45.

[25] Pham D.C., Shakedown theory for elastic plastic kinematic hardening bodies. International Journal of Plasticity 23 (2007) 1240-1259.

[26] Pham D.C., On shakedown theory for elastic-plastic materials and extensions. Journal of the Mechanics and Physics of Solids 56 (2008) 1905-1915.

[27] Pham D.C., On adaptation of elastic-perfectly plastic structures to dynamic loading cycles, Proceedings of the International Conference on Applied Dynamics, Hanoi-11/1995, 195-201.

[28] Pham D.C., A reduced form of shakedown kinematic theorem. Vietnam Journal of Mechanics 24, N.1, 25-34 (2002)

[29] Pham D.C., Modified Shakedown theorems for elastic-perfectly plastic structures. Proceedings of VII-National Conference on Mechanics of Solids, Do son 27-28/4/2004, pp. 58-64.

[30] Alexandrov, S, Pham, D.C. Effect of Singular Velocity Fields on Shakedown Analysis: A Simple Analytical Example, Proc. COMPLAS IX, E. Onate, D.R.J. Owen, B. Suarez (Eds.), Part 1, CIMNE, Barcelona, Spain, 2007, pp.174-177.

[31] Pham D.C., Shakedown and Collapse of Elastic-plastic Kinematic-hardening Structures. VIII-National Conference on Mechanics Hanoi- 12/2007, pp. 69-78.

Received May 5, 2009

\section{THÍCH NGHI VÀ HỎNG CỦA CÁC KẾT CẤU ĐÀN DẺO CHỊU TẢI TRỌNG THAY ĐỔI VÀ LẶP LẠI}

Bài viết giới thiệu một số kết quả chính tác giả nhận được trong phân tích thích nghi các kết cấu đàn dẻo, với các định lý thích nghi cho vật liệu đàn dẻo tái bền động học đóng vai trò trung tâm. Một số ví dụ áp dụng được trình bầy. 